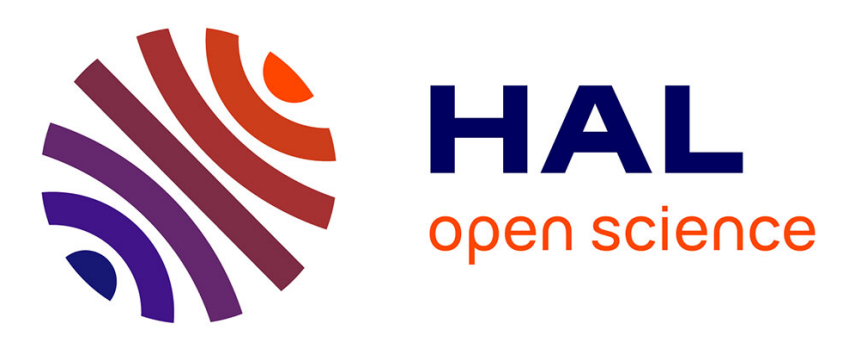

\title{
Criticality in the slowed-down boiling crisis at zero gravity
}

T Charignon, P Lloveras, D Chatain, L Truskinovsky, E Vives, D Beysens, Vadim Nikolayev

\section{- To cite this version:}

T Charignon, P Lloveras, D Chatain, L Truskinovsky, E Vives, et al.. Criticality in the slowed-down boiling crisis at zero gravity. Physical Review E , 2015, 91, pp.053007. 10.1103/PhysRevE.91.053007 . cea-01366768

\section{HAL Id: cea-01366768 https://hal-cea.archives-ouvertes.fr/cea-01366768}

Submitted on 15 Sep 2016

HAL is a multi-disciplinary open access archive for the deposit and dissemination of scientific research documents, whether they are published or not. The documents may come from teaching and research institutions in France or abroad, or from public or private research centers.
L'archive ouverte pluridisciplinaire $\mathbf{H A L}$, est destinée au dépôt et à la diffusion de documents scientifiques de niveau recherche, publiés ou non, émanant des établissements d'enseignement et de recherche français ou étrangers, des laboratoires publics ou privés. 


\title{
Criticality in the slowed-down boiling crisis at zero gravity
}

\author{
T. Charignon, ${ }^{1}$ P. Lloveras, ${ }^{2}$ D. Chatain, ${ }^{1}$ L. Truskinovsky, ${ }^{2}$ E. Vives,${ }^{3}$ D. Beysens,,${ }^{1,4}$ and V. S. Nikolayev ${ }^{5, *}$ \\ ${ }^{1}$ Service des Basses Températures, UMR-E CEA/UJF-Grenoble 1, INAC, 17 rue des Martyrs, 38054 Grenoble Cedex 9, France \\ ${ }^{2}$ Laboratoire de Mécanique des Solides, CNRS-UMR 7649, Ecole Polytechnique, Route de Saclay, 91128 Palaiseau, France \\ ${ }^{3}$ Departament d'Estructura i Constituents de la Matèria, Universitat de Barcelona Martí i Franquès 1, \\ Facultat de Física, 08028 Barcelona, Catalonia, Spain \\ ${ }^{4}$ Physique et Mécanique des Milieux Hétérogènes, UMR 7636 ESPCI, CNRS, Univ. Paris-Diderot, Univ. P.M. Curie, \\ 10 rue Vauquelin, 75005 Paris, France \\ ${ }^{5}$ Service de Physique de l'Etat Condensé, CNRS UMR 3680, IRAMIS/DSM/CEA Saclay, 91191 Gif-sur-Yvette, France
}

(Received 15 January 2015; published 12 May 2015)

\begin{abstract}
Boiling crisis is a transition between nucleate and film boiling. It occurs at a threshold value of the heat flux from the heater called CHF (critical heat flux). Usually, boiling crisis studies are hindered by the high CHF and short transition duration (below $1 \mathrm{~ms}$ ). Here we report on experiments in hydrogen near its liquid-vapor critical point, in which the $\mathrm{CHF}$ is low and the dynamics slow enough to be resolved. As under such conditions the surface tension is very small, the experiments are carried out in the reduced gravity to preserve the conventional bubble geometry. Weightlessness is created artificially in two-phase hydrogen by compensating gravity with magnetic forces. We were able to reveal the fractal structure of the contour of the percolating cluster of the dry areas at the heater that precedes the boiling crisis. We provide a direct statistical analysis of dry spot areas that confirms the boiling crisis at zero gravity as a scale-free phenomenon. It was observed that, in agreement with theoretical predictions, saturated boiling CHF tends to zero (within the precision of our thermal control system) in zero gravity, which suggests that the boiling crisis may be observed at any heat flux provided the experiment lasts long enough.
\end{abstract}

DOI: 10.1103/PhysRevE.91.053007

\section{INTRODUCTION}

When during boiling the supplied heat flux exceeds a critical value [the critical heat flux (CHF)], the vapor bubbles on the heating surface form abruptly a continuous vapor film that drastically reduces the heat transfer. The temperature of the heater rapidly grows, which can cause its melting. This is very dangerous for industrial heat exchangers, in particular in nuclear power plants. The transition is known under the names of "boiling crisis" (BC) and "departure from nucleate boiling." Correct CHF estimation requires a clear understanding of the physical phenomena that triggers it. However, the number of parameters and interactions involved in a quantitative description of boiling is so large that no definitive theory has been achieved yet. Since the discovery of BC [1], a large amount of both experimental data and theoretical models has been accumulated. On the one hand, the mechanisms governing the bubble formation, growth, and detachment, and their dependence on external parameters, are rather well understood [2]. On the other hand, triggering factors of the vapor film formation and the role of collective effects are still elusive.

During the past decade, advanced methods of observation and measurement (as high speed and high resolution optical and infrared cameras, microscopic thermal sensors and structured heating matrices, etc.) were applied to access experimentally the length scales smaller than the vapor bubbles and time scales below the bubble growth time. These methods considerably extend earlier studies $[3,4]$ and a consensus about the local nature of $\mathrm{BC}$ is presently emerging.

*Corresponding author: vadim.nikolayev@cea.fr
PACS number(s): 47.55.df, 64.60.av, 64.70.fh, 64.60.Ht

Contrary to the previous idea of $\mathrm{BC}$ as being triggered by bulk hydrodynamic phenomena [2], the phenomena responsible for the $\mathrm{BC}$ are believed to be acting in a thin fluid layer adjacent to the heater [5-14], down to the level of the lines of triple liquid-solid-vapor contact. The accent is made on the growth dynamics of dry heater area. It is important to understand how drying impacts the triggering of the BC. Either it is triggered by the growth of dry spots under individual vapor bubbles $[5,8,15,16]$ that coalesce later, or $\mathrm{BC}$ results from a collective phenomenon of the avalanchelike multiple bubble coalescence [17]. This is still a question requiring further investigation.

Growth of dry spots under individual bubbles is caused mainly by the increase of the apparent contact angle. For bubbles during boiling, the relation between the apparent contact angle and the size of the dry spot was demonstrated both experimentally [18] and by numerical simulation [15]. It has been shown $[8,19]$ that at near critical pressure, where the hydrodynamic flow is very slow, the vapor recoil is the main cause of the apparent contact angle increase as a result of its relative strength with respect to the capillary force (the contact angle is defined, as usual, at the liquid side). Farther away from the liquid-vapor critical point, the viscous pressure drop of the liquid flow toward the contact line is the main cause of the apparent contact angle increase [20,21].

In the past few years, different experimental approaches have suggested that the boiling crisis (BC) may be a scalefree phenomenon which implies criticality [22-29]. The appearance of $1 / f$ noise $[30,31]$ and the increase in magnitude of chaos quantifiers, like the fractal patterns of the wall temperature fluctuations [32] and of the outlines of the voids [33], are signatures of the absence of characteristic scales in the $\mathrm{BC}$ vicinity. 
The relevance of collective effects to $\mathrm{BC}$ has been strongly supported by recent acoustic emission experiments [17], that have revealed that the energy distribution of acoustic signals emitted by boiling becomes a power law close to the crisis. These observations suggest criticality and a simplified boiling model presented in Ref. [17] explains why the distribution of dry spot sizes exhibits subcritical-critical-supercritical transition. However, a complete experimental justification of the criticality in the BC is still unavailable. Subcritical-like distributions have been observed in previous experiments: nonboiling bubble columns [34] and weak partial nucleate boiling [35] have shown the Gaussian probability density distribution. Also, assuming circular dry spots, which is reasonable in nucleate boiling, Kim et al. [35] and Kim and Benton [36] observed enhancement of the mean size and the appearance of a long tail of the distribution when the heat flux was increased. This also might point out a tendency towards criticality. In Ref. [37], the authors present distributions close to the crisis and in the transition boiling regime. Although their distributions seem to follow a power law, the quality of fit remains poor. In general, efforts addressing the analysis of merging or spreading bubbles have been mostly restricted to one or few bubbles only. To complement these experiments, we carry out in this paper a first statistical analysis of the size distribution of dry spots and follow its evolution in time.

The violent boiling conditions under which $\mathrm{BC}$ occurs complicate a detailed analysis and hinder a deeper understanding of the process. Experimental studies at normal conditions are very difficult to perform $[7,12]$ since the required heat fluxes are huge, e.g., $\sim 3 \mathrm{MW} / \mathrm{m}^{2}$ for water at atmospheric pressure. The main difficulty of such observations is the highly nonequilibrium nature of the crisis, which devaluates methods based on the idea of equilibrium steady state. In addition, the $\mathrm{BC}$ characteristic time is very small, typically under $1 \mathrm{~ms}$.

In the liquid-vapor critical point vicinity, the thermal diffusivity tends to zero and the bubble growth controlled by it slows down. The liquid flow also slows down and the optical distortions associated with the strongly deformed vapor-liquid interfaces and turbulence disappear. In addition, the CHF becomes very small [8], thus facilitating the experimental implementation. However, the capillary length tends to vanish too. The vapor-liquid interfaces become flat and formation of the round bubbles (and thus nucleate boiling in its conventional sense) is not possible any more. Therefore, the reduced gravity conditions are needed to maintain a conventional boiling geometry. The individual bubble spreading under low gravity was studied experimentally by some of us $[8,19]$. In the previous BC experiment [8], the layer of liquid was so shallow that the vapor bubbles during their growth reached the liquid surface before being able to coalesce with each other. The objective of the present article is to study the boiling crisis in a deep liquid pool where collective aspects of bubble coalescence may be revealed.

\section{EXPERIMENTAL APPARATUS}

The experiment is carried out in the installation HYLDE (Hydrogen Levitation Device) $[8,38]$ which compensates the

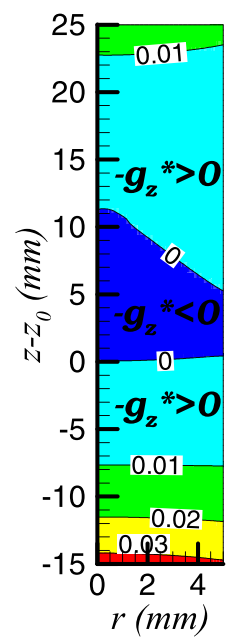

(a)

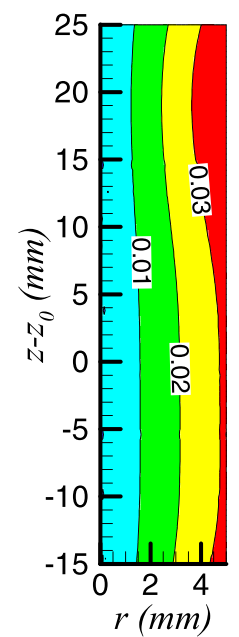

(b)

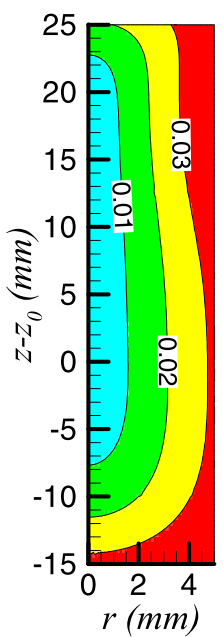

(c)
FIG. 1. (Color online) Residual gravity map calculated for HYLDE containing a ferromagnetic insert for $I_{h}=65.55 \mathrm{~A}$ of (a) axial, (b) radial components of the force $-\vec{g}^{*} / g$, and (c) its modulus $g^{*} / g$.

gravity in two phase (liquid and gas) hydrogen by magnetic force. The hydrogen is diamagnetic and its weight can be compensated with the magnetic force per unit volume [39-41]

$$
\vec{F}_{m}=\frac{\chi_{m}}{2 \mu_{0}} \vec{\nabla}\left(B^{2}\right),
$$

where $B$ is the magnetic field, $\mu_{0}$ the magnetic permeability of vacuum, and $\chi_{m}<0$ the magnetic susceptibility of $\mathrm{H}_{2}$. In the Earth gravity, the residual gravity acceleration (a force per unit mass acting on hydrogen) is

$$
\vec{g}^{*}=\vec{g}+\frac{\chi_{m}}{2 \mu_{0} \rho} \vec{\nabla}\left(B^{2}\right),
$$

where $\vec{g}$ is the Earth gravity acceleration. Note that the direction of the residual force acting on liquid coincides with $\vec{g}^{*}$, while the direction of the residual ("buoyancy") force acting on gas bubbles inside the liquid coincides with $-\vec{g}^{*}$. Since in boiling we deal with vapor bubbles, the residual gravity map is more convenient to present in terms of $-\vec{g}^{*}$ (Fig. 1).

Since $\chi_{m}$ is proportional to the density $\rho$ of a diamagnetic substance, $\alpha=\chi_{m} / \rho$ is independent of density. It means that the vertical buoyancy force is compensated if one applies the $B^{2}$ gradient $d B^{2} / d z=2 \mu_{0} g / \alpha$, where $z$ is the vertical upward coordinate. For $\mathrm{H}_{2}, 2 \mu_{0} g / \alpha \sim-941 \mathrm{~T}^{2} / \mathrm{m}$ and is independent of temperature.

It was demonstrated [42] that the magnetic force (1) cannot be made uniform in a volume. In HYLDE, Earth gravity can be exactly compensated at two points located at the coil axis (i.e., at $r=0$, where $r$ is the radial coordinate) near the top of the superconductive coil. In our case, the exact compensation point height is $z_{0}=71.5 \mathrm{~mm}$ above the coil center when the coil current is 65.55 A. For such a regime, Fig. 1 gives the calculated isovalues of the components and of the modulus of the residual forces (per unit mass) acting on a vapor bubble. They were calculated with the Radia freeware [43]. Inside the 
cell described below the residual gravity level does not exceed $3.3 \times 10^{-2} \mathrm{~g}$.

Unlike the paramagnetic case [44], the equilibrium of small gas bubbles located at both these points inside the liquid is unstable. At the upper compensation point [Fig. 1(a)], the gas bubble is unstable in the vertical direction. This can be easily checked [44] by analyzing the force acting on the bubble situating slightly above and below the compensation point. It is evident [Fig. 1(a)] that the forces bring the bubble away from the compensation point. This means that the bubble always moves out of this point, so that it cannot be used or even localized experimentally. The other exact compensation point corresponds to $z=z_{0}$. A gas bubble located at this point is stable in the axial direction. Indeed, if a bubble located initially at $z=z_{0}$ moves along the axis to the point $z>z_{0}$, the force acting on it is directed downwards [Fig. 1(a)]. Were the bubble moved downwards, the force would become upward, which would cause the bubble to return to its initial position again. Since the radial component of the force is positive at any $r \neq 0$ [Fig. 1(b)], the bubble is unstable in the radial direction. This means that a vapor bubble that situates initially near the coil axis tends to move away from it. To understand the phase distribution inside the cell (see below), it is important to note that the opposite stability conditions hold for the liquid drops inside the gas [44]. In particular, the liquid drop is radially stable (i.e., centered) at the $z=z_{0}$ point.

One needs to minimize the residual magnetic forces. To improve the force field homogeneity, a ferromagnetic ring of specially calculated shape can be inserted inside the coil [41]. It however reduces the useful bore into which the internal cryostat containing the experimental cell ("anticryostat") should fit. This cryostat is used to thermally insulate the coil immersed into the liquid $\mathrm{He}$ at $2.17 \mathrm{~K}$ from the cell at the $\mathrm{H}_{2}$ critical point temperature $T_{c} \simeq 33 \mathrm{~K}$. The compensation volume is about twice larger (for the same maximal residual acceleration) with insert comparing to the previous experiments [8] where the bare coil was used. As a result, experiments with a deeper pool of liquid are now possible.

When the $\mathrm{H}_{2}$ temperature $T$ approaches $T_{c}$, all physical properties of hydrogen depend strongly on the temperature difference $T-T_{c}$ that thus needs to be well controlled. However the $T_{c}$ value itself changes with time. At room temperature (i.e., before the experiment), $\mathrm{H}_{2}$ is a $3 / 1$ mixture of orthohydrogen (parallel spins of $\mathrm{H}$ nuclei, o- $\mathrm{H}_{2}$ ) with parahydrogen (antiparallel spins, $\mathrm{p}-\mathrm{H}_{2}$ ). When $\mathrm{H}_{2}$ is cooled down, the $\mathrm{o}-\mathrm{H}_{2}-\mathrm{p}-\mathrm{H}_{2}$ equilibrium is shifted and $\mathrm{o}-\mathrm{H}_{2}$ transforms gradually to $\mathrm{p}-\mathrm{H}_{2}$, which causes a $T_{c}$ decrease. This transformation is however very slow comparing to the change of $T$ that we impose during the experiment. The $T_{c}$ value follows an exponential law with the time constant of $2500 \mathrm{~min}$. During a week of measurements, which is the total duration of the present experiment, the $T_{c}$ decrease was about $0.1 \mathrm{~K}$. The $T_{c}$ value was measured several times a day and its current value was used to process each data set so that the $T-T_{c}$ difference was well defined. We checked that the magnetic-field induced $T_{c}$ shift (due to thermodynamic effect and magnetic-field influence to the Cernox ${ }^{\circledR}$ thermistors used for the temperature measurement) is insignificant.

One can distinguish two parts in the cell (Fig. 2). The upper part consists of a transparent sapphire tube and a metal

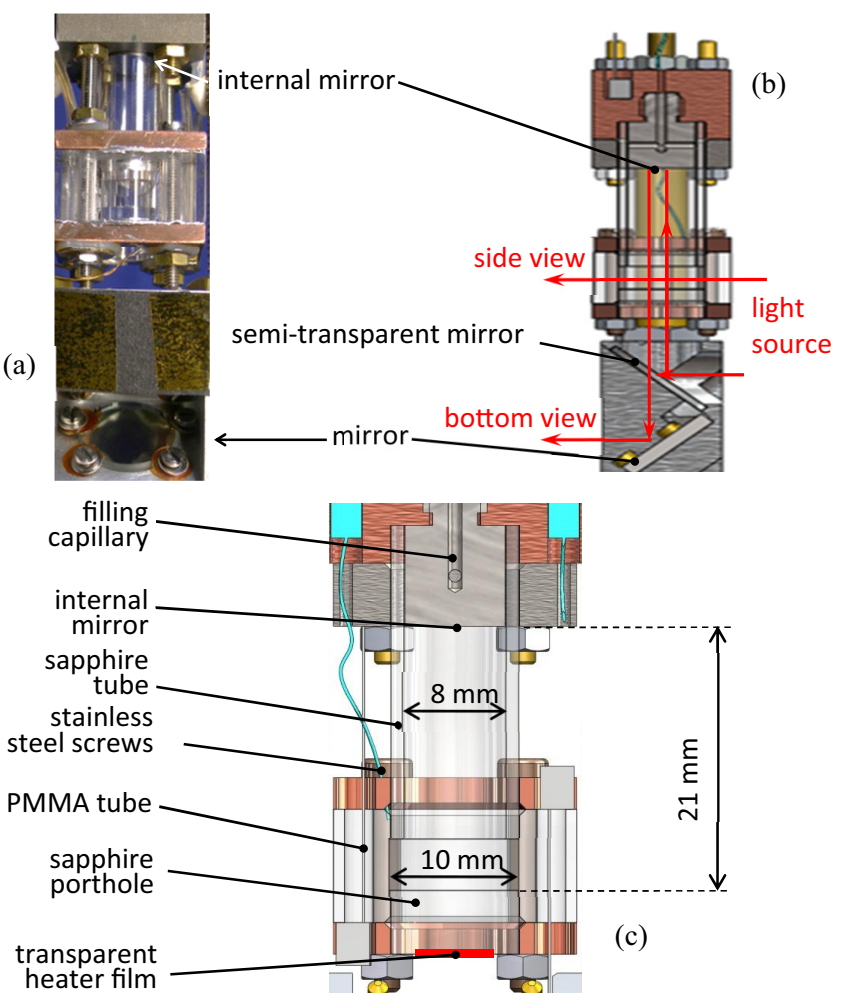

FIG. 2. (Color online) Experimental cell: (a) photo, (b) drawing showing two way optical cell observation paths, and (c) more detailed drawing of the cell alone.

flange. Its central area is polished and serves as an internal mirror for optical observations through the transparent heater. By using a system of mirrors [Fig. 2(b)] and movable with stepper motors endoscopes, the cell can be observed either from below (through the transparent heater) or sidewise. The sidewise observations are absolutely necessary, e.g., to find the point of exact gravity compensation. The observations are performed with a $1024 \times 1024$ CCD camera, 30 frames/s. A telescopic lens has to be used because the camera is situated at the top of the cryostat, at about $2 \mathrm{~m}$ from the cell [40]. Only a part of the image, of the size about $750 \times 750$ pixels, is useful; it is limited by the optical aperture.

The upper cell flange is cooled by a connection with the cold cryostat and incorporates two electrical heaters and thermistors. With their help, the upper flange temperature $T$ is stabilized with a PID controller within $0.1 \mathrm{mK}$. The flange has a good thermal contact with the sapphire tube due to the indium O-ring seal. Because of the excellent heat conductivity of sapphire at low temperatures and its good thermal contact with the liquid, the temperature $T$ corresponds to the bulk fluid temperature, i.e., the saturation temperature corresponding to the gas pressure. The lower part of the cell is the PMMA tube that connects the middle and lower copper flanges. The lower flange has a transparent $10 \mathrm{~mm}$ diameter sapphire porthole that serves as a local fluid heater [Fig. 2(c)]. The porthole itself is heated with a transparent metal oxide film of the same type as in the DECLIC [45] boiling cells. Its resistance at nominal regime is about $48 \Omega$. The heater is able to inject the heat power $P_{h}$ up to $350 \mathrm{~mW}$ that corresponds to the flux of 
$4.5 \mathrm{~kW} / \mathrm{m}^{2}$. The heater temperature $T_{h}$ is recorded. A middle flange that situates on the top of the PMMA tube is pressed against the lower part with four stainless steel screws. The connections between the tubes and the flanges are sealed with the indium O-rings. Due to the small thermal conductivity of PMMA $(0.088 \mathrm{~W} / \mathrm{m} / \mathrm{K})$ and stainless steel $(3.3 \mathrm{~W} / \mathrm{m} / \mathrm{K})$ at $33 \mathrm{~K}$, the thermal resistance between the lower flange and the rest of the cell is quite high $\left(R_{\mathrm{th}}=68 \mathrm{~K} / \mathrm{W}\right)$ so that the heat flux generated in the heater is fully supplied to the boiling fluid (except probably the film boiling regime [46] that is not considered here). Measurements of $R_{\text {th }}$ performed with the empty cell agree with the above theoretical value. Note that there are no other heat leaks from the heater because the cell is in vacuum and the mirror holder [below the cell in Fig. 2(a)] is attached to the upper flange of the cell; it does not touch the cell lower part. The thermal control of the cell is thus much better than in a previous study [8] by some of us, where a substantial amount of heat flux leaked via the cell lateral walls.

In the beginning of the experiment, the cell initially under vacuum is filled in situ with pure $\mathrm{H}_{2}$ through a capillary [Fig. 2(c)] until attaining an average density inside the cell corresponding to the $\mathrm{H}_{2}$ critical density. The capillary is then closed with a thermal cryogenic valve. The critical density is assumed to be attained when the liquid meniscus position at $1 g$ does not change when the temperature $T$ is slowly varied from $T_{c}-10 \mathrm{mK}$ toward $T_{c}$ until critical opalescence obscures the meniscus image. This method makes use of the symmetry of liquid-vapor coexistence curve in the $T_{c}$ vicinity with respect to the critical density. When the cell is filled at the critical density, the symmetry causes the liquid-vapor volume ratio in the cell to be unity, independently of $T$. After having cooled down the coil, each experiment begins with localizing $z_{0}$. Then the cell position is adjusted with respect to $z_{0}$ to provide the necessary level of gravity at the heater. Indeed, the whole anticryostat can be moved with a stepper motor inside the coil so that the position of the exact compensation point can be changed with respect to the cell.

\section{PHASE DISTRIBUTION IN THE CELL}

The shape of bubbles or drops is influenced by the residual magnetic forces when their size is larger than the corresponding capillary length $l^{*}=\sqrt{\sigma /\left(\Delta \rho g^{*}\right)}$ [38], where $\sigma$ is the surface tension and $\Delta \rho$ is the difference of liquid and gas densities. The value of $l^{*}$ increases with decreasing $g^{*}$. It remains however small. For instance, for $g^{*}=0.02 \mathrm{~g}$ [cf. Fig. 1(c)], $l^{*}$ is about $10 \mathrm{~mm}$ when $T=20 \mathrm{~K}$ corresponding to 1 bar saturation pressure. For $T_{c}-T=20 \mathrm{mK}, l^{*} \simeq 1 \mathrm{~mm}$. For the cell of $1 \mathrm{~cm}$ diameter described above, it means that the shape of small bubbles (like those nucleating and growing on the heater) is not influenced by the residual gravity and is thus spherical. However, the radius of curvature of the gas-liquid meniscus in the half-filled cell is much larger and the meniscus shape is thus impacted by the residual gravity. This influence is especially strong at small $T_{c}-T$, which is the most interesting regime because of the slowing down of $\mathrm{BC}$. It is discussed next.

Similar to [8], two main regimes can be distinguished at zero heating depending on $T_{c}-T$. Let us consider first a situation where the compensation point situates in the middle of the cell and the experiments are performed at progressively higher temperatures $T$ approaching $T_{c}$ from below. When $T_{c}-T$ is larger than a threshold value of about $0.5 \mathrm{~K}$, the phase distribution in the tube is controlled mainly by the capillarity: the gas forms a convex bubble surrounded by the liquid. Because of complete (critical) wetting, the cell walls are covered by liquid wetting film. At a smaller $T_{c}-T$, the residual magnetic forces become stronger than the surface tension. The bulk liquid forms a column (or elongated drop) along the cell axis by the action of the relatively strong radial forces [Fig. 1(b)] that push the gas to the walls. The liquid column in most cases touches only one base of cylindrical cell [Fig. 3(a)].

In the experiments described below, the residual gravity level is defined by the $g^{*}$ value at the location of the heating

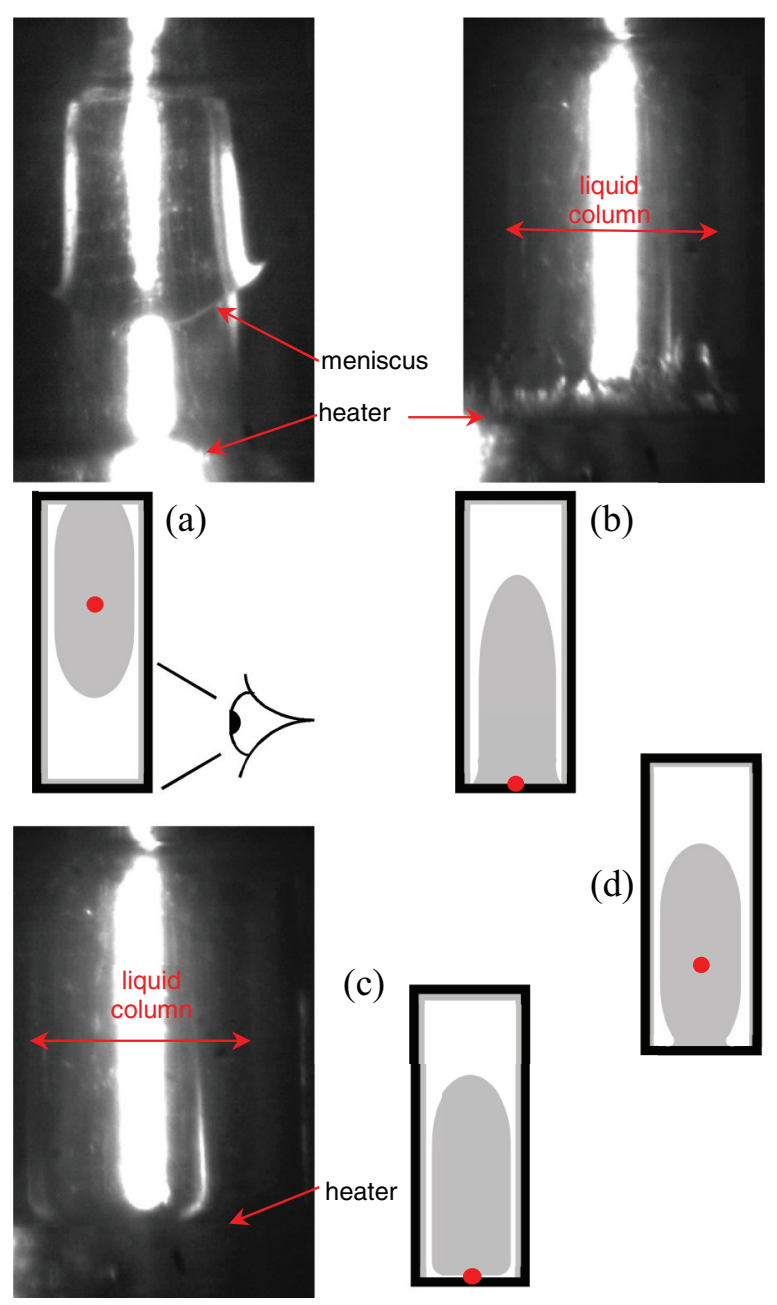

FIG. 3. (Color online) Photos of the phase distribution in the lower part of the cell at magnetic gravity compensation [(a)-(c), side view] and the corresponding sketches. The exact compensation point is shown in the sketches with a dot. The gray color corresponds to the liquid, while white indicates the gas. (a) Compensation point in the middle of the cell at zero heating; the residual gravity at the heater level is directed downward. The liquid column does not touch the heater. (b),(c) Compensation point is at the heater level during boiling ( $0 g$ situation): (b) before and (c) right after the boiling crisis. Figure (d) sketches a situation similar to (a) but with the column touching the heater. 
surface where bubbles nucleate and grow. When it situates in the exact compensation point $z=z_{0}$, such conditions are referred to as $0 g$. The bulk liquid covers the whole heater at $0 g$ [Fig. 3(b)] close to the critical point. Farther away, a small heater portion becomes uncovered because of the higher surface tension with respect to the magnetic forces.

\section{OBSERVATION OF THE BOILING PROCESS}

Each boiling run is performed at constant both $P_{h}$ and $T$. After the heating start, the heater temperature $T_{h}$ rises before becoming nearly constant [48]. The temperature rise decreases with the $T_{c}-T$ decrease and could not be detected below $T_{c}-T \sim 10 \mathrm{mK}$ (i.e., becomes smaller than $1 \mathrm{mK}$, which is a limitation imposed by the data acquisition system). At each run, when the CHF is attained, heating is stopped, the vapor film breaks, and the heater is rewetted by liquid. After the temperature equilibration at newly chosen $T$ value, the experiment is restarted with a newly chosen $P_{h}$.

The time constant of the $T_{h}$ rise is of the order of several seconds and corresponds to the balance of thermal inertia of the sapphire porthole and quite efficient nucleate boiling heat exchange. Sapphire is an excellent heat conductor with $5830 \mathrm{~W} \mathrm{~m}^{-1} \mathrm{~K}^{-1}$ thermal conductivity at $33 \mathrm{~K}$ and for this reason isothermal. Note that the heat conductivity of copper $1150 \mathrm{~W} \mathrm{~m}^{-1} \mathrm{~K}^{-1}$ is quite smaller. However, there are other characteristic times related to the physics of BC (probably, occurrence of a rare event of large dry area creation as a result of coalescence) so that the $\mathrm{BC}$ can occur tens of minutes after $P_{h}$ has been switched on.

Consider first the $0 g$ conditions (Fig. 4) where the whole heater is covered by the liquid column [cf. Fig. 3(b)] and the smallest exploitable $T_{c}-T=10 \mathrm{mK}$. The heater view is shown in Fig. 4; the size of snapshots is $8 \times 7.9 \mathrm{~mm}^{2}$ with a resolution of $750 \times 744$ pixels. Because of the almost nonexistent nucleation barrier, weak boiling starts with a very small heating power. In particular, several $\mu \mathrm{W}$ (according to our estimates) brought to the heater through the electrical wires connected to the metal oxide film (residual heat leak because of the wire heat conduction) were sufficient, so it existed even at $P_{h}=0$ [Fig. 4(a)]. When heating is applied, intense nucleate boiling starts. Far from dryout, boiling resembles a classical nucleate boiling pattern where the bubbles (and the dry spots) are round [Figs. 4(a)-4(c)]. Closer to the dryout [Figs. 4(d) and 4(e)], the bubbles lose their spherical shapes. The time between subsequent coalescences becomes smaller than the characteristic shape relaxation time equal to $\sim \sigma / \mu$ times the bubble size, with $\mu$, the liquid shear viscosity (the latter expression is justified at small Reynolds numbers). The bubbles cannot then restore their spherical shape between subsequent coalescences and keep all the time nonequilibrium complicated shapes. This is visible in Figs. 4(d) and 4(e) (see Supplemental Material [47]). At the time moment of dryout the porthole dries out completely; cf. Fig. 4(f). This shows clearly that the $\mathrm{BC}$ occurs through the chain coalescence of dry spots.

The bubbles do not leave the heater at $0 g$. Under the action of the residual gravity forces, they slide instead from the heater center towards the periphery, i.e., the liquid column lateral surface where they merge with the bulk vapor. In Fig. 3(b),
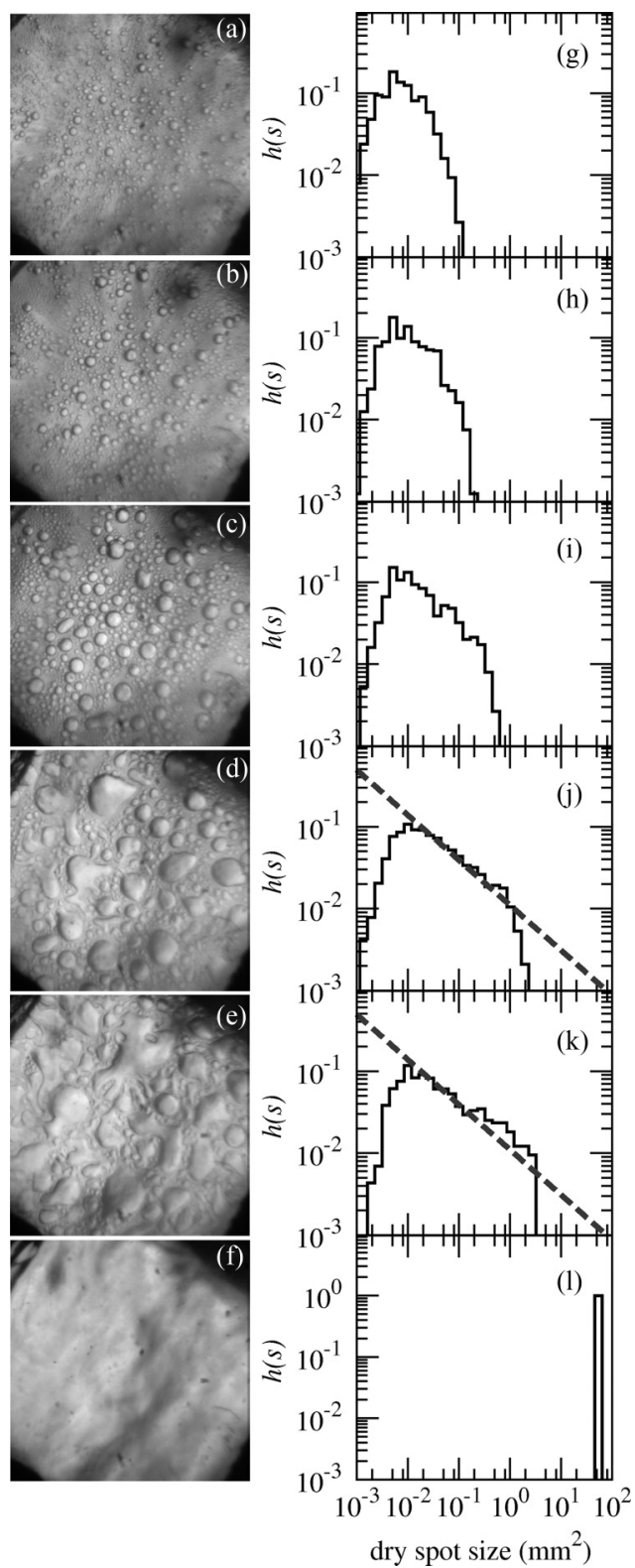

FIG. 4. Left column: boiling of $\mathrm{H}_{2}$ at $T_{c}-T=10 \mathrm{mK}$ and $0 g$ seen through the transparent heater. Images (a)-(c) correspond to the nucleate boiling far from $\mathrm{BC}$ at heater current $I_{h}=0,2$, and $5 \mathrm{~mA}$, respectively, whereas images (d)-(f) show the BC dynamics under $I_{h}=7 \mathrm{~mA}, 290 / 30$ th, 20/30th, and 0/30th s before the dryout, respectively (see Supplemental Material [47]). Right column: dry spot size distributions of the corresponding left-hand images. Dashed lines correspond to $h(s) \propto s^{-1.55}$.

one can see these bubbles inside the liquid column. Therefore, in spite of the absence of the vertical gravity component, the bubble residence time on the heater is limited by their sliding.

The dry area under each bubble expands with time (i.e., the contact lines recede) until the bubbles coalesce with its nearest neighbor. The contact line receding occurs because of the apparent contact angle increase caused by the strong evaporation (i.e., high heat flux) in the contact line vicinity [8,19-21]. These combined bubbles coalesce in their turn. 

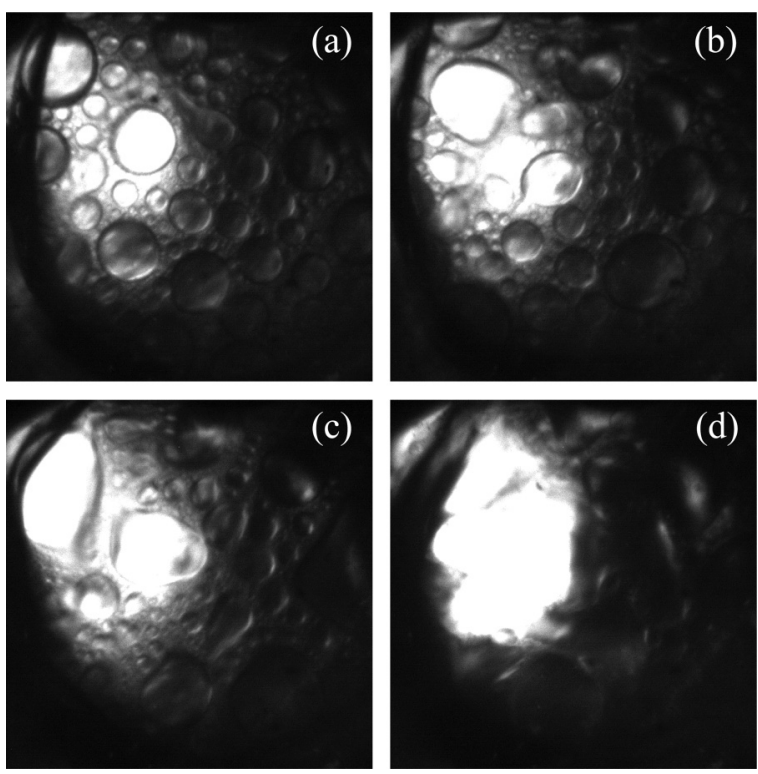

FIG. 5. Boiling crisis dynamics in $\mathrm{H}_{2}$ at $T_{c}-T=78 \mathrm{mK}, I_{h}=$ $19.5 \mathrm{~mA}$, and $0 g$ seen through the transparent heater. The images correspond to (a) $37 / 30$ th s, (b) $14 / 30$ th s, (c) 6/30th s, and (d) $1 / 30$ th s before the heater dryout.

The dry heater area keeps growing until the total heater dryout occurs. One needs to mention that the dry area growth should cause the growth of the heat flux that passes through the liquid. Indeed, the vapor has a higher thermal resistance than the liquid so that the heat flux is deviated to the latter.

According to the videos in the Supplemental Material [47] bubble coalescence occurs immediately after touching of the contact lines of neighboring bubbles. This feature shows that the apparent contact angles should remain right or obtuse. In the opposite case, the bubble merge would occur before the dry area merge. Therefore, the bubble size corresponds to the dry area size.

Farther from $T_{c}$, the bubble growth accelerates and the bubbles are mostly spherical. This occurs because the surface tension becomes larger. The coalescence-dominant regime before $\mathrm{BC}$ where the bubbles are nonspherical becomes very short and difficult to observe; cf. Fig. 5.

\section{ZERO CHF ISSUE}

$\mathrm{BC}$ is conventionally detected through two sudden simultaneous events: $T_{h}$ strong rise and disappearance of bubble nucleation at the heater indicating the heater dryout [cf. the transition between Figs. 4(e) and 4(f)]. In the present experiments, no threshold heat flux of $\mathrm{BC}$ (meaning zero CHF) has been detected. The CHF has been searched as follows. First, the boiling runs at progressively higher $P_{h}$ were carried out until BC occurred within $30 \mathrm{~s}$ from the power switching. The typical $P_{h}$ value for such an event increased with $T_{c}-T$. Such a behavior is in line with the preceding experiment [8] where the CHF was almost proportional to $T_{c}-T$.

After having reached $\mathrm{BC}$, boiling runs at progressively lower (but constant in time) $P_{h}$ were tried. With the $P_{h}$ decrease, the waiting time before $\mathrm{BC}$ strongly increased.
However, BC occurred inevitably. The experiments were stopped when waiting time exceeded a half an hour. At one occasion, we obtained CHF with $P_{h}=0$ (i.e., only with small uncontrollable heat leak) when the cell remained unintentionally under the field during several hours. The waiting time variation seemed to follow a power law of $P_{h}$ with a negative exponent (the exact law could not be established because of experimental time limitations). Such a behavior is not surprising since all the BC theories predict zero CHF at zero gravity. However, in all (to our knowledge) previous experiments, finite CHF values were found in microgravity. In experiment [8], this can be explained by the presence of a characteristic length corresponding to the thickness of liquid layer that limits the bubble maximum diameter, just like the capillary length does under finite gravity where CHF is finite. For the conventional (low pressure) boiling spatial experiments, CHF data are obtained rather accidentally than systematically [49] and for this reason cannot be considered as conclusive.

To see what happens at finite residual gravity level, another series of experiments has been performed at $g_{z}^{*}=-0.02 g$ at the heater. Such a gravity level has been obtained by placing the cell at $\cong 12 \mathrm{~mm}$ lower than the exact compensation point [at the position $z=z_{0}-12 \mathrm{~mm}$ in Fig. 1(a)]. Surprisingly, the $T_{h}$ dynamics observations also show zero CHF value, which contradicts the known experimental facts [2]. Such an anomalous behavior can be understood when analyzing the optical observations (Fig. 6).

When the exact compensation point is above the heater, the bulk liquid covers only the central heater part [cf. Fig. 3(d)]. The residual heat leak is sufficient to dry out completely the remaining heater surface. One detects the disappearance of wetting film on it by the absence of bubble nucleation. This means that there is a contact line that encircles the wetted heater area and situates $a t$ the heater. Together with the increase of the dry area below each bubble, the wetted heater area decreases with time (Fig. 6). The latter feature is absent in the $0 g$ case. Such a decrease explains the zero CHF because the heat flux through the liquid increases with time much faster than in the $0 g$ case thus causing further drying of the heater until its complete dryout. The total heater dryout has been obtained even with $P_{h}=0$, the residual heat leak being sufficient. Such a behavior qualitatively confirms the apparent contact angle increase with the heat load [21].

\section{DRY AREA GROWTH ANALYSIS}

The evolution of the dry area is studied below. The image processing for the detection of the dry spots areas has been carried out manually: the varying and inhomogeneous brightness makes the standard algorithms of the automatic image processing unsuccessful. Bubbles that go past the image frame are not included into the analysis.

Examples of the original picture and the corresponding processed image are shown in Figs. 7(a) and 7(b), respectively. After processing, size distributions $h(s)$ have been obtained by counting the number of dry spots with an area of $s \mathrm{~mm}^{2}$, and normalizing by the total number of dry spots. An undercounting of very small dry spot areas $s$ below 100 pixels $^{2}$ $\left(s \leqslant 0.01 \mathrm{~mm}^{2}\right.$ ) occurs due to the limited resolution. Separate 

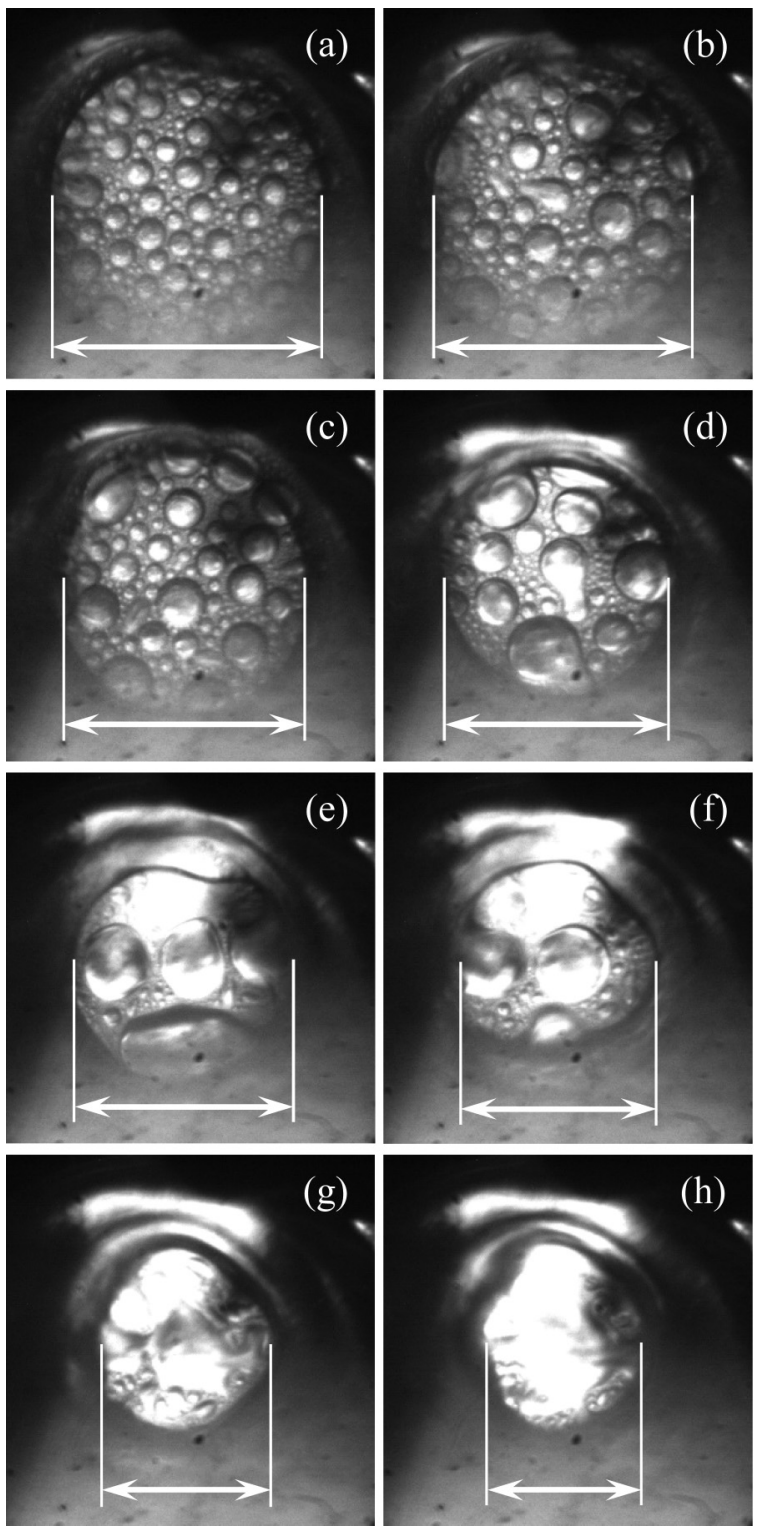

FIG. 6. Boiling crisis dynamics in $\mathrm{H}_{2}$ seen through the transparent heater at $T_{c}-T=17 \mathrm{mK}, I_{h}=7 \mathrm{~mA}$, and $0.02 \mathrm{~g}$. The images correspond to (a) 489/30th s, (b) 384/30th s, (c) 213/30th s, (d) $27 / 30$ th s, (e) 23/30th s, (f) 9/30th s, (g) 6/30th s, and (h) $4 / 30$ th $s$ before total heater dryout. The arrow shows the diameter of the portion of heater area in contact with the liquid that decreases with time.

analysis of the individual frames of the video confirms the existence of different regimes identified visually before. It is found that the distribution of dry spot sizes does not vary during the first $200 / 30 \simeq 6.7$ s. It corresponds to nucleate boiling far from dryout, which occurs at $315 / 30 \simeq 10.5 \mathrm{~s}$ of the video in the Supplemental Material [47] corresponding to Figs. 4(d)-4(f). This allows us to average the corresponding histograms within this time interval, thus improving the statistics [50]. The histograms have been averaged also in the interval $280 / 30-300 / 30 \simeq 9.3-10 \mathrm{~s}$, which corresponds to the coalescence-controlled evolution. The intermediate interval $200 / 30-280 / 30 \simeq 6.7-9.3 \mathrm{~s}$ has been discarded to

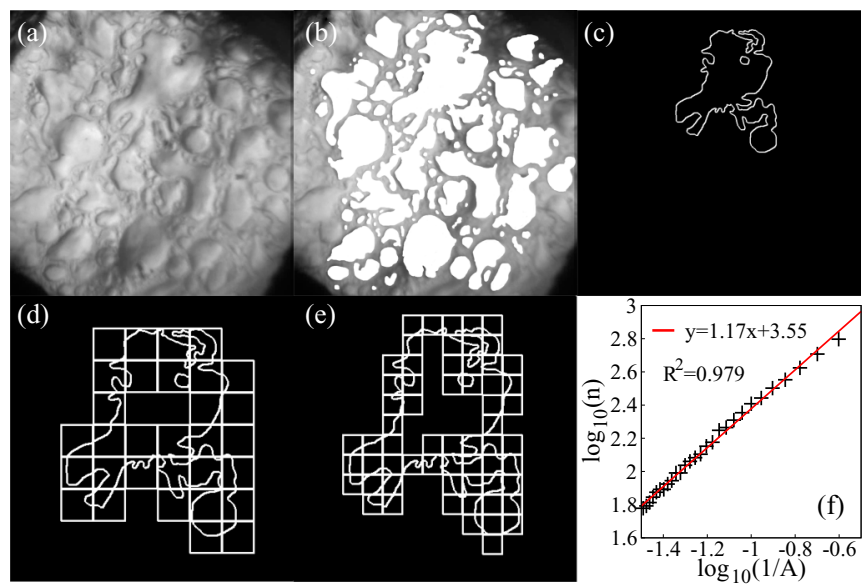

FIG. 7. (Color online) Image (a) and its corresponding processed snapshot (b) where white denotes vapor phase. In this case $I_{h}=7 \mathrm{~mA}$. Panels (c)-(f): box counting method. For more details, see the text.

avoid possible overlapping of regimes. For $t>300 / 30 \simeq 10 \mathrm{~s}$ the liquid-vapor interfaces become hard to distinguish.

In Fig. 4, to the right of each snapshot we show the associated averaged dry spot size distributions $h(s)$ in logarithmic scale. A clear exponentially damped decay is observed for large sizes [panels (g)-(i)]. This case corresponds to the conventional nucleate regime far from dryout showing the round dry spots. These data are comparable with previous experimental studies [37] showing the Gaussian-like distribution. The tail of distribution becomes heavier as the boiling intensity rises. At some intermediate point between panels $(j)$ and (k), the distribution becomes a power law with an exponent $\alpha=1.55 \pm 0.10$ (indicated by the dashed lines) calculated with the maximum likelihood method and tested with the Kolmogorov-Smirnoff distance method [51]. The power-law distribution indicates the absence of characteristic dry spot size. The distribution becomes single peaked at the system size right after dryout [panel (1)].

To further characterize the system evolution, some additional quantities have been calculated. In particular, the top left panel in Fig. 8 shows the evolution of the averaged density $\rho$ of dry spots (defined as the number of dry spots per $\mathrm{mm}^{2}$ ) with areas $s>0.011 \mathrm{~mm}^{2}$ (left axis) and $s>1.1 \mathrm{~mm}^{2}$ (right axis) for the case $I_{h}=7 \mathrm{~mA}$. The former threshold is used to discard a bias due to the undercounting of smaller dry spots. An averaged constant number is observed within the first $8 \mathrm{~s}$, and then decreases to finally reach the lowest value corresponding to the vapor film. The threshold $s>1.1 \mathrm{~mm}^{2}$ is used to highlight the appearance of very large dry spots and, interestingly, exhibits the opposite behavior. A low number of large dry spots is found up to $t=8 \mathrm{~s}$, then it rises significantly and finally drops due to the formation of the film. The top right panel in Fig. 8 shows the same variables in terms of the different boiling regimes, including the cases $I_{h}=0,2$, and $5 \mathrm{~mA}$. The $x$ axis in this case is arbitrarily scaled.

Vapor fraction and mean dry spot size are shown in the bottom left panels of Fig. 8 for the case $I_{h}=7 \mathrm{~mA}$ as a function of time. Again, both quantities are constant in average during the first $8 \mathrm{~s}$ and then rise. The behavior through the different 


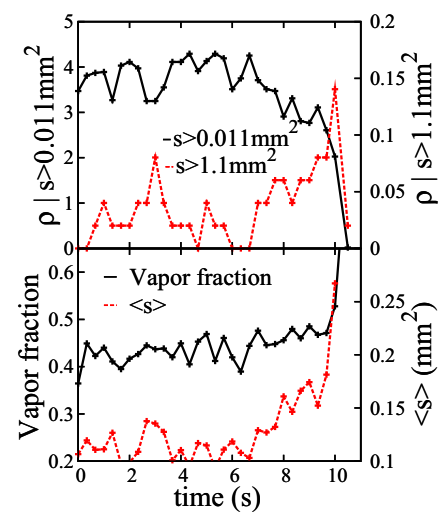

(a)

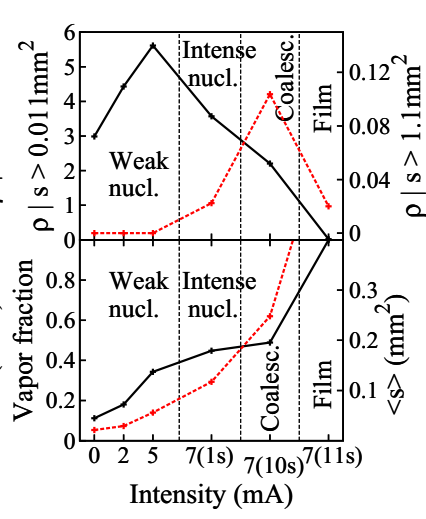

(b)
FIG. 8. (Color online) Top panels: density of the bubbles with size $s>0.011 \mathrm{~mm}^{2}$ (left axes, black solid curves) and $s>1.1 \mathrm{~mm}^{2}$ (right axes, red dotted curves) as functions of time (a) and boiling regime (b). Bottom panels: vapor fraction (left axes, black solid curves) and mean dry spot size (right axes, red dotted curves) as functions of time (a) and boiling regime (b). Panels (a) correspond to the case $I_{h}=7 \mathrm{~mA}$, whereas panels (b) also include the data for the nucleate boiling at $I_{h}=0,2$, and $5 \mathrm{~mA}$ [corresponding to Figs. 4(a)-4(c)].

regimes is displayed in the bottom right panel by including the cases $I_{h}=0,2$, and $5 \mathrm{~mA}$ of Fig. 4 .

\section{BOILING CRISIS AND CRITICALITY}

A typical signature of criticality is appearance of percolating clusters that exhibit fractal features [29]. In the BC context, a percolating cluster corresponds to the irreversible spanning dry spot that eventually covers the heater. Although some liquid-vapor interfaces cannot be clearly distinguished in the images, it is interesting to see how the fractal dimension of the contact lines evolves during the full boiling process, from the very beginning till the formation of the film. A priori it is expected that the periphery of round dry spots appearing well before the dryout has a fractal dimension close to unity and that it will increase for the ramified dry spots anticipating the film regime. For the computation of the fractal dimension, we use the box counting algorithm [33]. This method is illustrated in Figs. 7(c)-7(f). The snapshot 7(c) shows the profile of a selected dry spot size contained in snapshot 7(b). Figures 7(d) and 7(e) show the (magnified) boundary of a sample dry spot with superimposed boxes of decreasing sizes. Finally, Fig. 7(f) shows a number of boxes needed to cover entirely the dry spot perimeter as a function of the box size. A power law is indicative of self-similarity. Fitting the data by least squares we obtain the power-law exponent, which is directly associated with the box counting (or fractal) dimension (BCD). In this case, $\mathrm{BCD}=1.17 \pm 0.02$.

Looking for general trends in the BCD of the dry spots, we show in the left panel of Fig. 9 the dependence of BCD on the dry spot size. Averaging has been carried out (along the whole process) over bubbles of similar sizes (binning), with size intervals taken to vary logarithmically with the size. It can be seen that for very large dry spots (those that appear only close to dryout), BCD increases significantly, as expected.

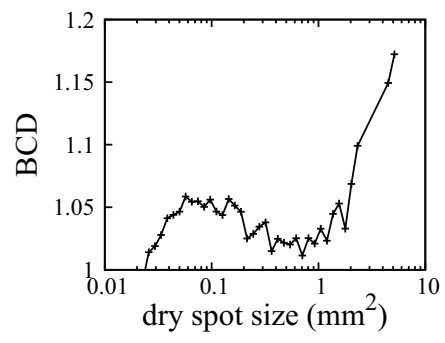

(a)

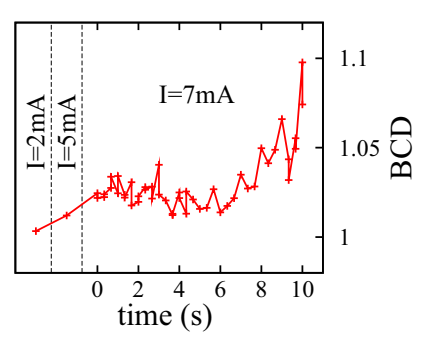

(b)
FIG. 9. (Color online) Box counting dimension (BCD) of dry spot perimeters for the experiment at $I_{h}=7 \mathrm{~mA}$ corresponding to Figs. 4(d)-4(f). (a) BCD averaged over the whole evolution as a function of the dry spot area. (b) BCD averaged over dry spots area $s>0.011 \mathrm{~mm}^{2}$ as a function of time. Data for the experiments at $I_{h}=2 \mathrm{~mA}$ and $5 \mathrm{~mA}$ [i.e., for the snapshots shown in Figs. 4(b) and 4(c)] are also included.

The right panel of Fig. 9 shows the time variation of the BCD calculated taking into account the dry spots with sizes $s>0.011 \mathrm{~mm}^{2}$. We observe an increasing trend of the BCD when approaching the dryout that confirms the scale-free behavior during the BC [17]. The observed trend resembles critical percolation [29].

\section{CONCLUSION}

We report optical observations of the boiling crisis dynamics slowed down by a close vicinity of the vapor-liquid critical point. Slow dynamics allow us to observe in detail the evolution of dry spots under the vapor bubbles at the heater and to quantify the kinetics of the dry spot coalescence. Reduced gravity conditions are necessary to preserve the conventional bubble topology. Within the experimental accuracy, it has been shown that the critical heat flux of boiling crisis is zero under such conditions (zero gravity), which means that the boiling crisis occurs at any heat flux, provided the observation time is sufficiently long. Two dry spot evolution regimes have been identified. Long before dryout, the dry spots are almost round. Their size probability distribution is close to Gaussian (subcritical). Close to the dryout event, the avalanches of dry spot coalescence occur; the contour of the dry area becomes fractal and the dry spot size distribution becomes a power law. These observations are similar to the behavior of systems exhibiting criticality, in particular, the critical clusters show a lot of similarity with spanning clusters in percolation processes. The duration of this coalescence-controlled collective regime depends crucially on the closeness to the liquid-vapor critical point. It is sufficiently long for a detailed observation only in a very close vicinity of the critical point $(\leqslant 10 \mathrm{mK})$. Our results place the boiling crisis into the context of critical phenomena apparently corroborating previous acoustic measurements [17]. However, one still cannot exclude that the critical (i.e., scale-free) dynamics of dry spots is affected by the zero gravity conditions when the main length scale of the problem, the bubble departure diameter, tends to infinity. Because of limitation of gravitation compensation quality in the HYLDE installation, experiments at nonzero gravity could not be performed. Future experiments are planned in higher magnetic fields to achieve better compensation quality. 
The beginning of bubble coalescence coincides with the touching of the triple contact lines of the neighboring dry spots, which suggests that the apparent liquid contact angle is right or obtuse. This observation agrees with the experiments of another group [14] and corroborates the hypothesis of some of us [16] stating that the $90^{\circ}$ apparent contact angle is a threshold for the boiling crisis triggering. From these results, the importance of the dry spot coalescence in the boiling crisis dynamics is evident. However, additional experimental studies [52] are needed to capture the details of the transition from the subcritical (round dry spots) to the critical (fractal dry spot contours) regimes.

\section{ACKNOWLEDGMENTS}

The financial support of ANR of France (Project ALICE, ANR-08-BLAN-0212) is acknowledged. This work was supported by the MINECO (Spain), Project No. MAT2013-40590$P$. We thank CNES for the support in the framework of the "Fundamental and applied microgravity" (MFA) program. The SBT team is grateful to J. Chartier and to P. Bonnay for the HYLDE technical support and to the colleagues from ICMCB (Y. Garrabos, C. Lecoutre, and J.-P. Manaud) for the metal-oxide film deposition. V.N. thanks E. de Malmazet and D. Hitz for fruitful discussions.
[1] S. Nukiyama, J. Soc. Mech. Eng. Jpn. 37, 367 (1934) [translated in Int. J. Heat Mass Transfer 9, 1419 (1966)].

[2] V. K. Dhir, Annu. Rev. Fluid Mech. 30, 365 (1998).

[3] K. Torikai, K. Suzuki, and M. Yamaguchi, JSME Int. J. Ser. II 34, 195 (1991), http://ci.nii.ac.jp/naid/110002493376/en/

[4] H. J. van Ouwerkerk, Int. J. Heat Mass Transfer 15, 25 (1972).

[5] V. S. Nikolayev and D. A. Beysens, Europhys. Lett. 47, 345 (1999).

[6] S. G. Kandlikar, J. Heat Transfer 123, 1071 (2001).

[7] T. G. Theofanous, T. N. Dinh, J. P. Tu, and A. T. Dinh, Exp. Thermal Fluid Sci. 26, 793 (2002).

[8] V. S. Nikolayev, D. Chatain, Y. Garrabos, and D. Beysens, Phys. Rev. Lett. 97, 184503 (2006).

[9] H. J. Chung and H. C. No, Int. J. Heat Mass Transfer 50, 2944 (2007).

[10] I.-C. Chu, H. C. No, and C.-H. Song, Int. J. Heat Mass Transfer 62, 142 (2013).

[11] S. Gong, W. Ma, and H. Gu, Int. J. Heat Mass Transfer 79, 694 (2014).

[12] J. Jung, S. J. Kim, and J. Kim, J. Heat Transfer 136, 041501 (2014).

[13] V. V. Yagov, Int. J. Heat Mass Transfer 73, 265 (2014).

[14] O. Kannengieser, W. Bergez, and C. Colin, in Proceedings of the 15th International Heat Transfer Conference, Kyoto, Japan, 2014, paper IHTC15-8856.

[15] V. S. Nikolayev, D. A. Beysens, G.-L. Lagier, and J. Hegseth, Int. J. Heat Mass Transfer 44, 3499 (2001).

[16] V. Janeček and V. Nikolayev, Interf. Phenom. Heat Transfer, doi: 10.1615/InterfacPhenomHeatTransfer.2015012273

[17] P. Lloveras, F. Salvat-Pujol, L. Truskinovsky, and E. Vives, Phys. Rev. Lett. 108, 215701 (2012).

[18] S. G. Kandlikar and M. E. Steinke, Int. J. Heat Mass Transfer 45, 3771 (2002).

[19] Y. Garrabos, C. Lecoutre-Chabot, J. Hegseth, V. S. Nikolayev, D. Beysens, and J.-P. Delville, Phys. Rev. E 64, 051602 (2001).

[20] V. S. Nikolayev, Phys. Fluids 22, 082105 (2010).

[21] V. Janeček and V. S. Nikolayev, Phys. Rev. E 87, 012404 (2013).

[22] J. P. Sethna, K. Dahmen, S. Kartha, J. A. Krumhansl, B. W. Roberts, and J. D. Shore, Phys. Rev. Lett. 70, 3347 (1993).
[23] J. P. Sethna, K. A. Dahmen, and C. R. Myers, Nature (London) 410, 242 (2001).

[24] O. Kinouchi and M. Copelli, Nat. Phys. 2, 348 (2006).

[25] A. Corral, C. J. Pérez, and A. Díaz-Guilera, Phys. Rev. Lett. 78, 1492 (1997).

[26] L. Gil and D. Sornette, Phys. Rev. Lett. 76, 3991 (1996).

[27] E. Vives and A. Planes, Phys. Rev. B 63, 134431 (2001).

[28] J. A. Bonachela and M. A. Muñoz, J. Stat. Mech. (2009) P09009.

[29] D. L. Turcotte, Rep. Prog. Phys. 62, 1377 (1999).

[30] V. Koverda and V. Skokov, Physica A 262, 376 (1999).

[31] V. Skokov, V. Koverda, A. Reshetnikov, V. Skripov, N. Mazheiko, and A. Vinogradov, Int. J. Heat Mass Transfer 46, 1879 (2003).

[32] M. Shoji, Int. J. Heat Mass Transfer 47, 1105 (2004).

[33] V. Sathyamurthi, D. Banerjee, H. Sakamoto, and J. Kim, Int. J. Heat Fluid Flow 29, 207 (2008).

[34] R. Schäfer, C. Merten, and G. Eigenberger, Exp. Therm. Fluid Sci. 26, 595 (2002).

[35] J. H. Kim, K. N. Rainey, S. M. You, and J. Y. Pak, J. Heat Transfer 124, 500 (2002).

[36] J. Kim and J. F. Benton, Int. J. Heat Fluid Flow 23, 497 (2002).

[37] S. Nishio, T. Gotoh, and N. Nagai, Int. J. Heat Mass Transfer 41, 3191 (1998).

[38] R. Wunenburger, D. Chatain, Y. Garrabos, and D. Beysens, Phys. Rev. E 62, 469 (2000)

[39] E. Beaugnon and R. Tournier, J. Phys. III 1, 1423 (1991).

[40] D. Chatain and V. S. Nikolayev, Cryogenics 42, 253 (2002).

[41] V. Nikolayev, D. Chatain, D. Beysens, and G. Pichavant, Micrograv. Sci. Technol. 23, 113 (2011).

[42] L. Quettier, H. Félice, A. Mailfert, D. Chatain, and D. Beysens, Eur. Phys. J. Appl. Phys. 32, 167 (2005).

[43] O. Chubar, P. Elleaume, and J. Chavanne, J. Synchrotron Rad. 5, 481 (1998), see http://www.esrf.eu/Accelerators/Groups/ InsertionDevices/Software/Radia

[44] G. Pichavant, B. Cariteau, D. Chatain, V. Nikolayev, and D. Beysens, Micrograv. Sci. Technol. 21, 129 (2009).

[45] Y. Garrabos, C. Lecoutre, D. Beysens, V. Nikolayev, S. Barde, G. Pont, and B. Zappoli, Acta Astronaut. 66, 760 (2010).

[46] D. Chatain, C. Mariette, V. S. Nikolayev, and D. Beysens, Phys. Rev. E 88, 013004 (2013).

[47] See Supplemental Material at http://link.aps.org/supplemental/ 10.1103/PhysRevE.91.053007 for the full video. 
[48] V. S. Nikolayev, D. Chatain, and D. Beysens, in Proceedings of the 6th International Conference on Boiling Heat Transfer, Spoleto, Italy, 2006.

[49] J. Straub, Adv. Heat Transfer 35, 57 (2001).

[50] To minimize correlations, only one of every ten frames has been analyzed.

[51] A. Clauset, C. Shalizi, and M. Newman, SIAM Rev. 51, 661 (2009).
[52] V. Nikolayev, Y. Garrabos, C. Lecoutre, D. Hitz, D. Chatain, R. Guillaument, V. Janeček, and D. Beysens, in Proceedings of the 8th European Conference on Aerothermodynamics for Space Vehicles, ESA, Lisbon, Portugal, 2015, see http://www.congrexprojects.com/ Custom/15A01/Papers/Room\%201.1/Wednesday/Flow\%20 phenomena\%20on\%20propulsion\%20systems\%20I/90933_ Nikolayev.pdf 